\title{
Transmission Electron Microscopic and First-principles Study of $\mathrm{SrTiO}_{3} / \mathrm{GaAs}$ Hetero-interfaces
}

\author{
Liang Hong, Serdar Öğüt and Robert Klie \\ Department of Physics, University of Illinois at Chicago, Chicago, IL 60607
}

This $\mathrm{SrTiO}_{3} / \mathrm{GaAs}$ interface is primarily being studied due to the interest of using it in metal-oxidesemiconductor field-effect transistors (MOSFETs), where the GaAs substrate would act as the semiinsulating base material and the $\mathrm{SrTiO}_{3}$ would act as the barrier oxide layer between the GaAs and the gate material. Previously, $\mathrm{SrTiO}_{3}$ ultra-thin films on As-terminated $\mathrm{GaAs}$ substrate have been studied experimentally [1-3]. In this work, various configurations of the $\mathrm{SrTiO}_{3} / \mathrm{GaAs}$ interface are considered and characterized using transmission electron microscope (TEM), atomic-resolution Z-contrast imaging and Energy-dispersive X-ray spectroscopy (XEDS), along with first-principles-based density functional theory (DFT) calculations, to study the energetically most favorable configuration and obtain a deep understanding of the structural and electronic properties.

The sample used in this work is grown using molecular beam epitaxy (MBE) method. A $3 \mathrm{~nm} \mathrm{SrTiO}_{3}$ thin film is grown on Si substrate, and covered by a $1 \mu \mathrm{m}$ thick GaAs layer. The cross-sectional atomicresolution Z-contrast image (Figure 1) of the $\mathrm{SrTiO}_{3} / \mathrm{GaAs}$ interface is obtained using JEOL JEM-3010 TEM at $300 \mathrm{kV}$. The chemical components are characterized by XEDS. In Figure 1, we can see the typical dumbbell-structure of the GaAs [110] and the $\mathrm{SrTiO}_{3}$ [100] thin film. A higher resolution Zcontrast image will be obtained from JEOL ARM200CF scanning transmission electron microscopy (STEM) along with XEDS mapping to know the atomic structure and chemical components of the interface in the future work.

First-principles calculations are carried out within the framework of DFT using the projector augmented wave (PAW) method as implemented in the Vienna Ab initio Simulation Package (VASP) code with the generalized gradient approximation (GGA) in the scheme proposed by Perdew-Burke-Ernzerhof (PBE). The optimized lattice constant of bulk GaAs (Zinc-blende structure) and $\mathrm{SrTiO}_{3}$ (perovskite structure) are $5.76 \AA$ and $3.94 \AA$, respectively. For the thin $\mathrm{SrTiO}_{3}$ film on $\mathrm{GaAs}$ substrate, the lattice constant of $\mathrm{SrTiO}_{3}$ is set as $4.07 \AA$ to match the GaAs lattice parameters. Double-slab structural model with an $8 \AA$ vacuum in the middle is used to study the $\mathrm{SrTiO}_{3} / \mathrm{GaAs}_{\text {a }}$ hetero-interface (shown in Figure 2). With regard to stoichiometric and non-stoichiometric $\mathrm{SrTiO}_{3}$ terminated with either $\mathrm{SrO}$ or $\mathrm{TiO}_{2}$ layer in contact with either Ga or As surface, as well as different bonding directions, 32 different configurations of the $\mathrm{SrTiO}_{3} / \mathrm{GaAs}$ interface are considered. The formation energy of the interfacial configurations is calculated through the equation

$$
E_{\mathrm{f}}=E_{\mathrm{slab}}-n_{\mathrm{Ga}} \mu_{\mathrm{Ga}}-n_{\mathrm{As}} \mu_{\mathrm{As}}-n_{\mathrm{SrO}} \mu_{\mathrm{SrO}}-n_{\mathrm{TiO} 2} \mu_{\mathrm{TiO} 2} .
$$

The chemical potential for each component in the equation is constrained by their bulk chemical potentials correspondingly. By comparing the formation energy, non-stoichiometric $\mathrm{SrTiO}_{3}$ sandwiched by $\mathrm{SrO}$ layers are found to be favored in both $\mathrm{Ga}$ and As terminated situations. A phase diagram of formation energy comparison for all the interfacial configurations is shown in Figure 3. Nonstoichiometric $\mathrm{Ga} / \mathrm{SrO}$ interface is found to be energetically most favorable since it occupies the largest area in the phase diagram. 
References:

[1] R. F. Klie et al, Appl. Phys. Lett. 87 (2005) 143106.

[2] Q. Qiao et al, Phys. Rev. B. 85 (2012) 165406.

[3] Y. Liang et al, Appl. Phys. Lett. 85 (2004) 1217.

[4] This work was supported by the National Science Foundation (Grant No. DMR-1408427).
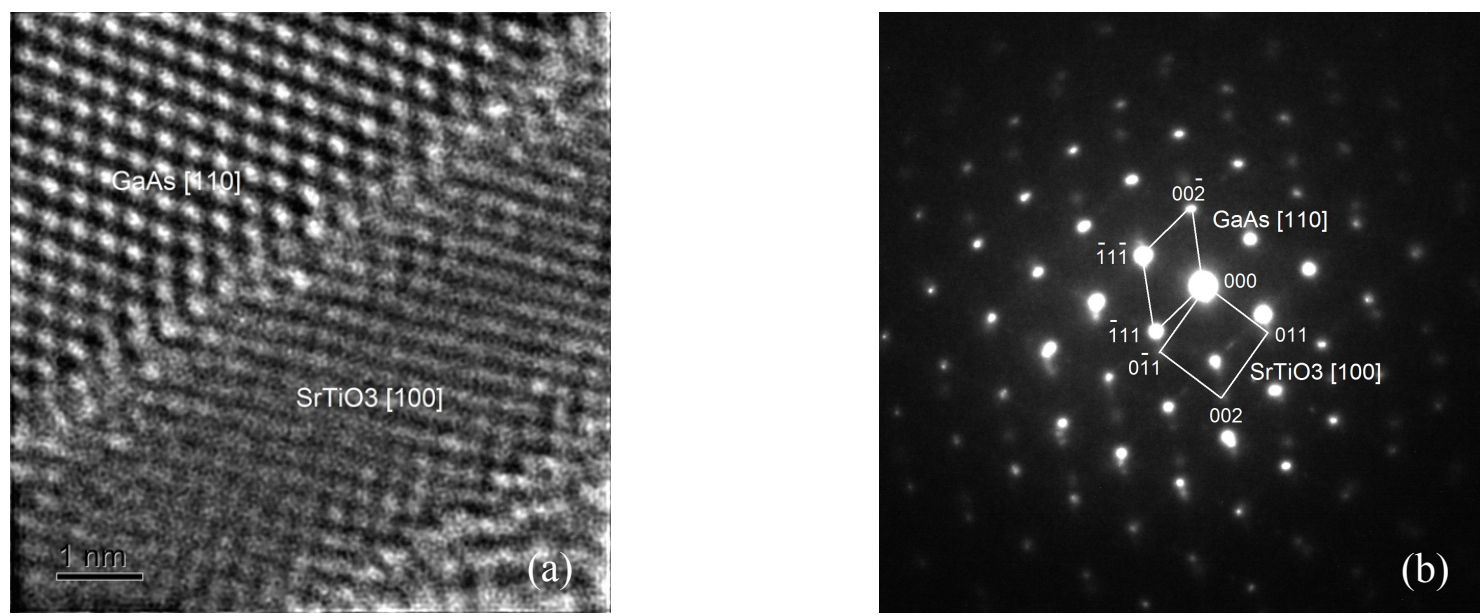

Figure 1 (a) Atomic-resolution Z-contrast image of the $\mathrm{SrTiO}_{3} / \mathrm{GaAs}$ hetero-interface. The GaAs is seen in [110] projection while $\mathrm{SrTiO}_{3}$ is in [100] projection. (b) Diffraction pattern on this condition.

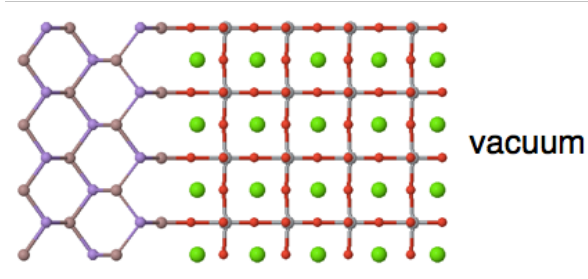

GaAs

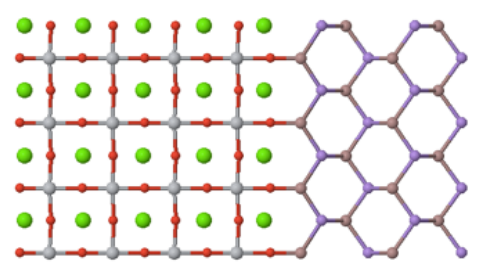

$\mathrm{SrTiO}_{3}$

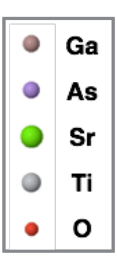

GaAs

Figure 2 Double-slab structural model used in the DFT calculations. A non-stoichiometric $\mathrm{SrTiO}_{3}$ terminated with $\mathrm{SrO}$ monolayer on the Ga-terminated GaAs substrate is shown in the figure.

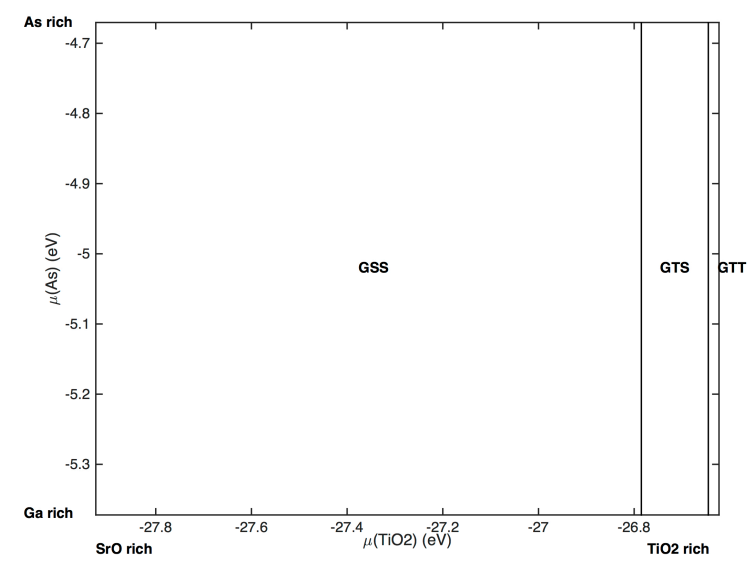

Figure 3 Phase diagram of the formation energy for the interfacial configurations. GSS, GTS, GTT represent the non-stoichiometric $\mathrm{Ga} / \mathrm{SrO}$, stoichiometric $\mathrm{Ga} / \mathrm{TiO}_{2}$ and non-stoichiometric $\mathrm{Ga} / \mathrm{TiO}_{2}$ interfaces, respectively. 